\title{
MuTaTo ${ }^{\circledR}$-A Novel Concept for Curing Cancer
}

\author{
Ilan Morad*, Hanan Itzhaki \\ Accelerated Evolution Biotechnologies (AEBi) Ltd., Nes-Ziona, Israel \\ Email: ^ilan@aebi-bio.com
}

How to cite this paper: Morad, I. and Itzhaki, H. (2020) MuTaTo ${ }^{\oplus}$ - A Novel Concept for Curing Cancer. Journal of Cancer Therapy, 11, 55-73.

https://doi.org/10.4236/jct.2020.112006

Received: November 27, 2019

Accepted: February 18, 2020

Published: February 21, 2020

Copyright (c) 2020 by author(s) and Scientific Research Publishing Inc. This work is licensed under the Creative Commons Attribution International License (CC BY 4.0).

http://creativecommons.org/licenses/by/4.0/ (c) (i) Open Access

\begin{abstract}
One of the main reasons for developing cancer drug-resistance is the ability of cancer cells to adopt mutations that help them fight the treatments. Cancer cells are very mutagenic. This makes the population of cancer cells in any tumor, or any other cancer cells that come from a distinct origin (one parent cell) highly variable. In some cases, there are already drug-resistant cancer cells at the beginning of the treatment. In other cases, they emerge during the treatment. Treating cancer patients with drugs, or other treatments that attack only one cancer-target would therefore be prone to bad prognosis. In addition, these kinds of treatments would also attack (to a lower degree) non-cancer cells that contain the same targets as the cancer cells. This would lead to adverse effects. Combination therapies, or bispecific drugs could partly solve these problems, but not completely. To address this and other problems, a novel concept for curing cancer, $\mathrm{MuTaTo}^{\oplus}$, was developed. MuTaTo is a personalized medicine concept. The main principal of it is using multiple targeting peptides connected together with a toxin. The main advantage of MuTaTo is that it would lower the probability of the targeted cancer cells to develop drug-resistance due to mutations they possess, and at the same time would lower adverse effects due to avidity effect. Each cancer patient would receive a specific MuTaTo drug perfectly suited to his cancer, based on the expression profile of receptors on the outer membrane of his cancer cells. MuTaTo construct production is easy and rapid. Therefore, the production cost would not be as expensive as with other biological drugs, or other sophisticated cancer treatments. In this article several experiments were performed to show the efficacy of different MuTaTo constructs, and the sustainability of the principals of this concept. The results showed that multi-targeting was better than mono targeting, and that MuTaTo was efficient as a mono treatment in vitro, and in vivo.
\end{abstract}

\section{Keywords}

MuTaTo, Multiple Targeting, Avidity Effect, Toxin, Curing Cancer 


\section{Introduction}

In 2017 cancer was the second most common death causing disease worldwide after cardiovascular diseases. 9.56 million people died of cancer during this year [1]. There are many kinds of cancer treatments. The main ones are surgery, chemotherapy, radiation therapy, hormonal therapy, immune therapy and targeted therapy. Cancer survival rates vary by the type of cancer, stage at diagnosis, treatment given and many other factors. In general, survival rates are improving over the years, but there is still a long way before cancer would be a curable disease. There are several reasons for poor prognosis in cancer drug-treatments. We can divide them into six categories:

1) Removal or destruction of drugs by our physiological systems before they reach their targeted cancer cells.

2) Access problems into tumors, or into the cancer cells.

3) Removal or destruction of drugs by various mechanisms of the cancer cells.

4) Activation of alternative signaling pathways and evasion of cell death.

5) Mutations in the cancer cells that disrupt the interaction with the drugs.

6) Cancer cells, or cancer promoting cells, like cancer stem cells, which are not targeted by the drugs.

Drug-resistance can be intrinsic (that is, present before treatment), or acquired during treatment by various therapy-induced adaptive responses [2]. In addition to the problems mentioned above, many cancer treatments are accompanied by serious adverse-effects that cause suffering to the patients, and sometimes make them give up the treatments [3]. An effective anti-cancer treatment should address all or most of the problems mentioned above.

$\operatorname{MuTaTo}^{\oplus}$ (Multi Target Toxin; Figure 1) is a novel personalized medicine concept of curing cancer patients. It is based on several principles that together would fight cancer drug resistance, with minimal side-effects:

1) Multiple targeting: attacking at least three cancer targets simultaneously.

2) Trojan Horse strategy: attaching a toxin to the drug. When the drug internalizes, the cancer cell gets a "poison peel", which destroys it. The toxin is inert outside of the cells.

3) Flexibility: the drug is flexible, and therefore would be able to penetrate difficult solid tumors.

4) No defined solid structure: the drug would be comprised of small peptides and a flexible scaffold that lacks a defined solid structure, and therefore would minimize the possibility of inducing an immune response [4].

5) Avidity effect: all the components of the drug would be connected. This would increase the interaction level of the drug with the cancer cells exponentially, and hence increase the efficacy substantially [5]. This effect would enable a decrease in the dose administration, while still achieving the desired therapeutic effect; lowering the dose would lead to lower adverse-effects. These adverse-effects are caused by interactions of the drugs with non-cancer cells that usually express lower amounts of the same cancer targets, and in most cases would not have the same combination of them. 


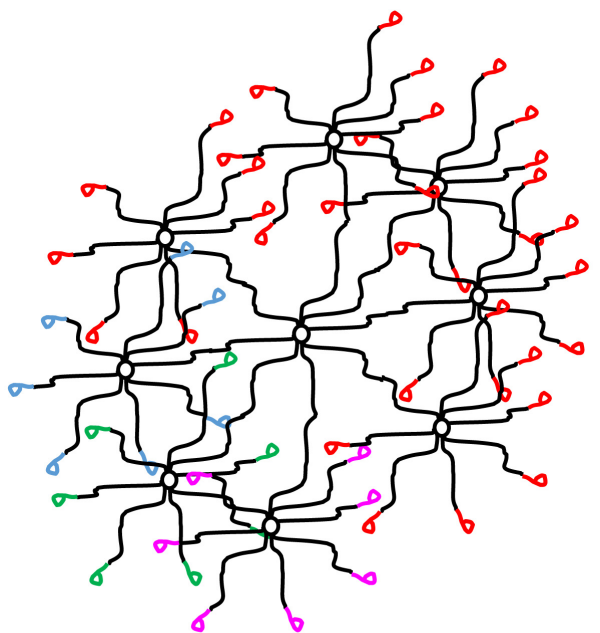

(a)

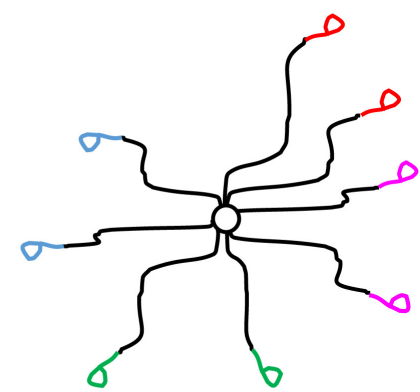

(b)

Figure 1. Illustration of $\mathrm{MuTaTo}^{\odot}$ (Multi Target Toxin) constructs. Targeting peptides are in blue, green and pink. Toxic peptides are in red. (a) 56-arm MuTaTo. (b) 8-arm MuTaTo.

Figure 2 describes a model of a cancer cell with receptors that are overexpressed on its outer membrane. When each one of these receptors is activated, it sends a message through the signal transduction pathway. This signal causes the cell to start many activities that contribute to the cancerous nature of the cell. When an antibody-drug $(\mathrm{Ab})$ targets, and interacts with this receptor, it inhibits it. By doing so, it stops the signal transduction, and in many cases leads to the cell's death (in some cases it also induces antibody-dependent cellular cytotoxicity (ADCC), antibody-dependent cellular phagocytosis (ADCP), and complement-dependent cytotoxicity (CDC)). There are many such drugs which are very successful. Examples include trastuzumab $\left(\right.$ Herceptin $\left.^{\oplus}\right)$, which is approved to treat certain breast and stomach cancers that overexpress HER-2; Cetuximab (Erbitux ${ }^{\otimes}$ ), which is approved to treat certain lung and colorectal cancers, and Squamous cell carcinoma of the head and neck that overexpress EGFR ; Rituximab (Ri$\operatorname{tuxan}^{\oplus}$ ), which is approved to treat non-Hodgkin's lymphoma, and chronic lymphocytic leukemia that overexpress CD-20.

When a cancer cell has a mutation in one of the proteins in the signal transduction pathway that makes it constitutively active, the receptor's activation is not needed, because the signal starts downstream. In this case, the antibody would not be able to stop the signal [6]. A common practice is to check for the presence of such internal mutations before giving these drugs to patients. If they do, the patients would not be treated with the drug, because it would be useless. These kinds of mutations could be acquired by the cancer cells during or after the treatment, if they are not destroyed completely. In this case, it would look like the treatment succeeded, but after a while it would come back, this time with drug-resistance. Another mutation could occur in the receptor itself, in such a way that would disable the interaction between the receptor and the antibody. Again, in this case the antibody would become non-effective. 


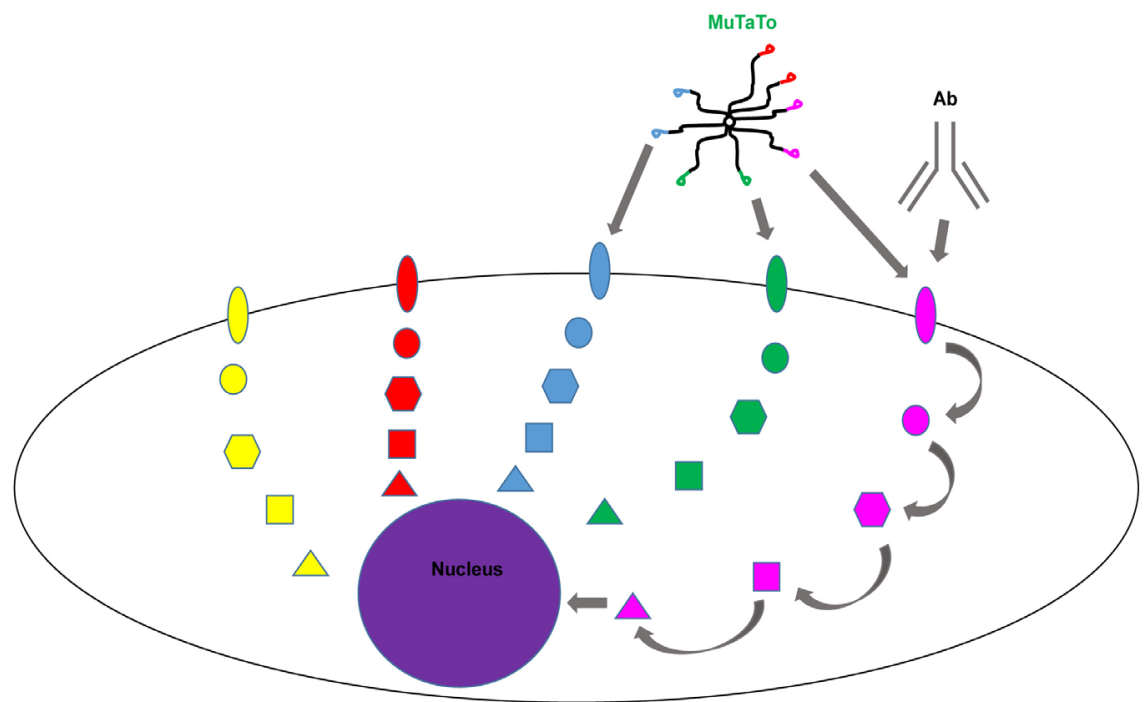

Figure 2. Illustration of a cancer cell, and two drugs attacking it. Proteins in the different signal transduction pathways, and their targeting peptides within MuTaTo are in pink, green, blue, red, and yellow. The antibody $(\mathrm{Ab})$ interacts with only one receptor. The MuTaTo interacts with three receptors.

There are a few more problems that can occur: In some cases, the antibody can have access problems entering solid tumors because of its large size and rigidity [7]. For the same reasons, antibodies (even humanized ones) can induce an immune response that will make them not useful anymore, due to their clearance from our blood by our own immune system.

To address these problems, a novel concept, $\mathrm{MuTaTo}^{\circ}$, was developed.

MuTaTo constructs, which are shown in Figure 1, are comprised of a multi-arm flexible scaffold. On the tip of each arm there is a small peptide. These peptides are divided into two categories: targeting peptides, and toxic peptides. Each MuTaTo molecule contains at least three kinds of targeting peptides that aim for different targets on the surface of the cancer cell. When these peptides interact with their targets (mostly receptors) they do two things: first, they inhibit them, and second, they get into the cell (internalization, endocytosis), together with the whole MuTaTo and the receptor. It is sufficient that one peptide interacts with its receptor to enable this process. The toxic peptides are not toxic outside the cells. This is because their targets are inside the cells, and they can't penetrate the cells by themselves. But when an internalization occurs due to the targeting peptides, these toxic peptides are able to interact with their target, inhibit it, and lead to cell death. The target of the toxic peptide could be a general essential target that exists in any cell, or a cancer-specific one.

The cancer cells of each patient would be analyzed using the same targeting peptides that are used in MuTaTos. Quantitative analysis of the receptors and their expression and response levels in the cancer cells would enable to fit the best personalized MuTaTo for the treatment of each cancer patient.

The MuTaTos in Figure 2 contain three kinds of targeting peptides. Down- 
stream mutation in the signal transduction pathway would not influence the effectiveness of the MuTaTo, because the internalization is not influenced by them, and the cancer cells should be destroyed by the toxic peptides, which act by a different mechanism. If a disabling mutation occurs in one receptor, there would still be two to work through; if two disabling mutations would occur simultaneously in two receptors, there would still be one to work through. Only if the cancer cell acquires three specific mutations simultaneously, then the specific MuTaTo would be useless. Such a triple mutation occurring simultaneously is so rare that it actually hardly ever happens. These mutations have to be new, because preexisting mutations in specific receptors would not lead to the inclusion of their targeting peptides in the specific MuTaTos.

This scenario is similar to what has happened with AIDS patients. When the patients used to be treated with one drug at a time, it helped for a little while, until the virus mutated, and then the drug was not useful anymore. The only treatment that succeeded was the cocktail, and now we already have HIV carriers who have been living for more than 20 years without being AIDS patients, and they will not become sick for the rest of their lives as long as they keep taking the cocktail; they would be HIV-1 carriers, but not AIDS patients [8].

Adverse-effects are caused by two mechanisms: 1) non-specific interactions of the drug with non-involved groups. 2) specific interactions of the drug with groups that exist in non-involved cells or tissues. In the second case, even the most specific antibody, which interacts with its target on the cancer cells, also interacts with the same target on non-cancer cells in our body. In most cases, the level of expression of the target is much higher in the cancer cells, and therefore most of the activity of the drug would be directed there, but the low level interaction with other cells could give serious adverse-effects [3].

Figure 3 explains how, by using the avidity-effect, the adverse-effects could be decreased. It describes a model of three cancer cells, and one which is not. The first cell (from left to right) overexpresses three kinds of cancer receptor targets on its surface. The second overexpresses two kinds of cancer targets, and the third overexpresses only one kind. The fourth cell expresses one type of receptor, and to a smaller extent. The MuTaTo in Figure 3 contains three targeting peptides to these three receptors. It would be very specific to the cancer cells that overexpress all three. It would be less specific to the cells that overexpress only two, and even less specific to the cell that overexpress only one, and the list specific to the cells that express only a small amount of one kind of receptor. Killing the cells that overexpress all three receptors, would require only a small amount of the MuTaTo. Killing the cells that overexpress only two, would require a higher concentration. Killing the cells that overexpresses only one, would require a further increase in the concentration administered. In order to kill all of the cells, except the ones that express only one of the receptors to a small extent (on the right), the concentration used should be in the range marked in orange. There should be a correlation between the efficacy and specificity of the drug, 


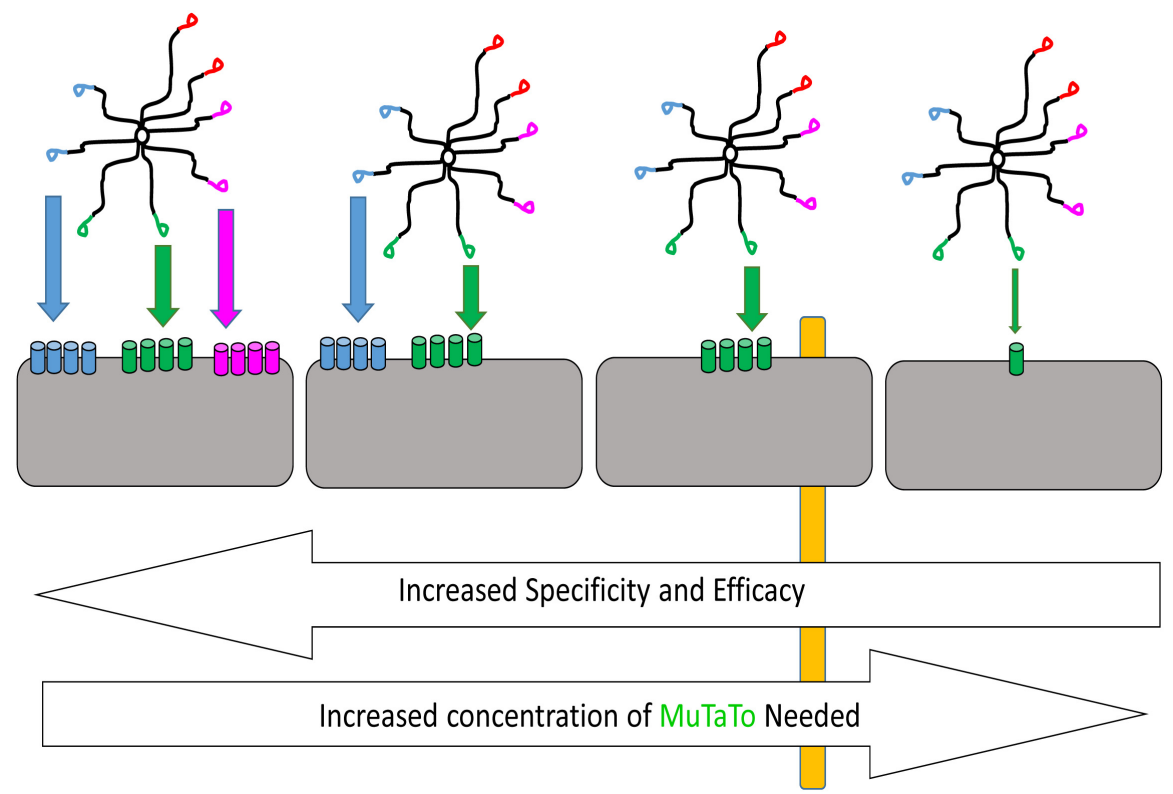

Figure 3. Illustration of three cancer cells, and one which is not (on the right). Different kinds of targeting peptides and their targeted receptors are in blue, green and pink. Toxic peptides are in red. The first cell (from left to right) overexpresses three kinds of cancer receptor-targets on its surface. The second overexpresses two kinds of cancer targets, and the third overexpresses only one kind. The fourth cell expresses one type of receptor, and to a smaller extent. The illustrated MuTaTos contain three targeting peptides to these three receptors.

and the number of potential interactions between the drug and the cancer cell. The interactions between the targeting peptides and the cancer cell do not have to be simultaneous. This is the mechanism of avidity [9].

There is another benefit of MuTaTo: two of the problems of cancer are cancer stem cells and metastasis. Many times the treatment kills the primary cancer cells but not the cancer stem cells and metastasis. The cancer stem cells are the originators of the primary cancer cells; therefore, they must be very similar to them. So, by the model described in Figure 3, the cell on the left represents a primary cancer cell, and the one on its right could represent the cancer stem cell. If this is the case, the cancer stem cells would be killed in the same treatment together with the primary cancer cells. The same logic goes with metastasis, which must be very similar to the primary cancer cells. So, in one treatment, the primary cancer cells would be killed together with the cancer stem cells and metastasis, and all other cancer cells that are similar to them.

In this paper we give the results of in vitro and in vivo experiments that were performed with different MuTaTo constructs. All the constructs contained a multi-arm PEG (Polyethylene glycol) attached to targeting and toxic peptides. Most of the peptides were discovered using our screening platform technology SoAP (https://patentscope.wipo.int/search/en/detail.jsf?docId=WO2007010525). The experiments demonstrate the efficacy of MuTaTo, and the validity of different principals of this concept. 


\section{Materials and Methods}

\subsection{Materials}

\subsubsection{Peptides}

See Table 1.

Table 1. Peptides.

\begin{tabular}{|c|c|c|}
\hline Name & Sequence & Target \\
\hline E13.3(dde) & $\begin{array}{l}\text { Ac-CHPGDK(dde)QEDPNCLQADK } \\
\text { cyclic by C-C bond }\end{array}$ & EGFR \\
\hline E13.3(fmoc) & $\begin{array}{l}\text { Ac-CHPGDK(fmoc)QEDPNCLQADK } \\
\text { cyclic by C-C bond }\end{array}$ & EGFR \\
\hline GR & $\begin{array}{l}\text { CEGLPADWAAAC } \\
\text { cyclic by C-C bond }\end{array}$ & PDL1 \\
\hline M582 & LSEGLGELMQRC & cMet \\
\hline GW & $\begin{array}{l}\text { CSARWGPTMPWC } \\
\text { cyclic by C-C bond }\end{array}$ & eEF2 \\
\hline $\mathrm{TB}$ & $\begin{array}{l}\text { CRRGSRASGAHC } \\
\text { cyclic by C-C bond }\end{array}$ & eEF2 \\
\hline GW2 & $\begin{array}{l}\text { CSARWGPIMPWC } \\
\text { cyclic by C-C bond }\end{array}$ & eEF2 \\
\hline BIM & MRPEIWIAQELRRIGDEFNE & Mitochondria \\
\hline
\end{tabular}

All peptides were produced in LifeTein LLC., NJ, USA.

\subsubsection{Chemicals}

8arm (TP) PEG Succinimidyl Carboxymethyl Ester, MW 73,000 was purchased from JENKEM TECHNOLOGY USA INC. TX, USA.

8arm (TP) PEG Succinimidyl Succinate MW 10,000 was purchased from Creative PEGWorks. NC, USA.

Dimethyl sulfoxide (DMSO), Dimethylformamide (DMF), Ethyl acetate, Fluorescein isothiocyanate (FITC), Triethylamine (TEA), formaldehyde, Hydrazine hydrate $(78 \%-82 \%), 1,4$ dioxane, Fmoc-Lys-OH were purchased from Sigma-Aldrich.

Phosphate-buffered saline (PBS) was purchased from BIO-LAB, Jerusalem, Israel.

\subsubsection{Ultrafiltration Apparatus}

Vivaspin 20, PES concentrator was purchased from Sartorius.

\subsubsection{Cancer Cell Lines}

A431, Human epidermoid carcinoma (ATCC CRL-1555'); NCI-H1650 Human non-small cell lung carcinoma, (ATCC CRL-5883); A549, Human epithelial lung carcinoma (ATCC, CCL-185); MCF-7 human epithelial adenocarcinoma, mammary gland, breast; derived from metastatic site: pleural effusion (ATCC HTB-22 $2^{\text {Tx }}$. 
Cultures were propagated at $37^{\circ} \mathrm{C} \pm 1^{\circ} \mathrm{C}$, humidified, $5 \% \pm 0.5 \% \mathrm{CO}_{2} /$ air, in plastic flasks. DMEM Medium (Biological Industries, Israel) supplemented with $2 \mathrm{mM}$ L-Glutamine, 10\% FBS (Fetal Bovine Serum), $1 \mathrm{mM}$ Sodium Pyruvate, $100 \mathrm{U} / \mathrm{ml}$ Penicillin and $100 \mu \mathrm{g} / \mathrm{ml}$ Streptomycin.

\subsection{Methods}

\subsubsection{Preparation of 56-Arm MuTaTo}

Each construct contained 56 peptides: 7 copies of each targeting peptide, and all the rest were toxic peptides. Peptides were dissolved in DMSO. 8arm (TP) PEG Succinimidyl Succinate, MW 10,000 was dissolved in 1,4-dioxane. Fmoc-Lys-OH was dissolved in $0.1 \mathrm{~N} \mathrm{HCl}$. Each peptide solution was mixed with Fmoc-Lys-OH solution and PEG solution at a molar ratio of 7:1:1 respectively. Triethylamine (TEA) was added to a final concentration of $5 \%$. The mixtures were incubated overnight at room temperature. Each mixture, which contained one kind of peptide, was combined with the other mixtures at a ratio that gave the desired molar ratio and combination of the peptides. PEG solution was added at a molar ratio of $1: 8$ to the PEG in the peptides mixture. The mixture was incubated for 2 hours at room temperature. When one of the peptides contained a dde protection group, hydrazine hydrate was added to a final concentration of $5 \%$, and the mixture was incubated for 2 hours at room temperature. The mixture was mixed with PBS at a final volume ratio of at least 1:20 respectively, and ultrafiltrated with vivaspin 20 concentrator of $30 \mathrm{kD}$ MWCO.

\subsubsection{Preparation of Fluorescent 8-Arm MuTaTo-Like Construct, Containing Anti-EGFR Peptides}

$17.6 \mathrm{mg}$ of Ac-E13.3 (fmoc) peptide was dissolved in DMSO to concentration of $10 \mathrm{mM}$ and mixed with 8arm (TP)PEG Succinimidyl Carboxymethyl Ester, MW 73,000 in 1,4 dioxane at a molar ratio of 16:1 respectively. TAE was added to a final volume concertation of $5 \%$. The mixture was incubated overnight at room temperature. $20 \mathrm{mM}$ FITC in DMF was added at a molar ratio of 1:2 peptide:FITC. The mixture was incubated for 3 hours at room temperature. The mixture was extracted twice with water saturated ethyl acetate, and the ethyl acetate traces were evaporated for 30 minutes with SpeedVac at $30^{\circ} \mathrm{C}$. The mixture was mixed with PBS at a final volume ratio of at least 1:20 respectively, and ultrafiltrated with vivaspin 20 concentrator of $10 \mathrm{kD}$ MWCO.

\subsubsection{Cell Growth and Viability Assay}

Different cancer cell lines from ATCC were thawed and cultivated to achieve exponentially growing cultures. Cells were collected, counted and seeded in a 96 well tissue culture plate at desired densities (2500 - 5000 cells/well). Plates were incubated until the next day at $37^{\circ} \mathrm{C} \pm 1{ }^{\circ} \mathrm{C}$, humidified, $5 \% \pm 0.5 \% \mathrm{CO}_{2} /$ air, to enable cells adherence to the wells.

At the next day, Growth Media were replaced with Test Items Solutions prepared in Assay Medium (2\%f FBS). Test Items Solutions are applied carefully (onto the sides of the well, not directly onto the cells) in volume of $200 \mu \mathrm{l} /$ well to 
achieve the final concentrations of the Test Items. Plates were incubated at $37^{\circ} \mathrm{C} \pm$ $1^{\circ} \mathrm{C}$, humidified $5 \% \pm 0.5 \% \mathrm{CO}_{2}$ /air. After 48 hours of incubation, representative images of cells treatments were taken on microscope. In some cases, the media in wells were replaced with fresh Growth Medium containing 10\% of AlamarBlue $^{\circledR}$ solution. Fluorescent signal (Excitation $544 \mathrm{~nm} /$ Emission $590 \mathrm{~nm}$ ) is measured following approximately 7 hours of incubation with Fluorescence Plate Reader Fluoroskan Ascent FL, Labsystems.

The $\mathrm{P}$ values were determined using T-Test in http://www.graphpad.com/quickcalcs/ttest1.cfm.

\subsubsection{Xenograft Mice Experiment}

Hsd:Athymic Nude-Foxn $1^{\text {nu }}$ mice, female, 6 - 7 weeks of age at tumor induction, obtained from accredited breeder. The tumor cell suspension (A549, Human epithelial lung carcinoma (ATCC, CCL-185); was injected to $n=12$ animals at dose volume of $0.2 \mathrm{ml} / \mathrm{An}\left(5 \times 10^{6}\right.$ cells/An. $)$ by a single SC injection into the right flank area, midway between the axillary and inguinal regions.

The Test Item and the Control Item were injected to $n=6$ tumor-bearing mice (initial tumor size of $\sim 80 \mathrm{~mm}^{3}$ and up) per group by the IV route, at dose volume of $4 \mathrm{ml} / \mathrm{kg}$. Test materials are injected 9 times (on weekdays only) during a period of three weeks (every 2 - 3 days).

Monitoring of progressive changes in tumor growth were carried out in all animals twice a week from measurable tumors until study termination, using Electronic Digital Calipers. The tumor volume is determined and calculated according to the following equation: $\mathrm{V}\left(\mathrm{mm}^{3}\right)=\mathrm{d}^{2}\left(\mathrm{~mm}^{2}\right) \times \mathrm{D}(\mathrm{mm}) / 2$. The symbols $\mathrm{d}$ and $\mathrm{D}$ represent the smallest and the largest perpendicular tumor diameters, respectively.

Viability checks, for mice mortality and morbidity, were performed at least once daily. Cage-side observation for the detection of abnormalities were also performed once daily. Whenever an abnormality was detected it was recorded. Determination of individual body weights of animals were made shortly before tumor induction (Day 0) and twice weekly thereafter.

The experiment protocol was in accordance with the standard animal welfare guidelines, and under the permission of Ethics Committee.

\subsubsection{PK Experiment in Mice}

Hsd:Athymic Nude-Foxn $1^{\text {nu }}$ mice, female , 6 - 7 weeks of age at tumor induction, obtained from accredited breeder. The tumor cell suspension (NCI-H1650 Human non-small cell lung carcinoma, ATCC CRL-5883) was injected to $n=5$ animals at dose volume of $0.2 \mathrm{ml} / \mathrm{An}\left(5 \times 10^{6}\right.$ cells/An.) by a single SC injection into the right flank area, midway between the axillary and inguinal regions. When the tumor size was of $\sim 50 \mathrm{~mm}^{3}, 0.075 \mathrm{ml}$ of PEG-E13.3-Fluorescein solutions were injected into one of the tail veins.

At different time points $(0,5,60,240,360,1440 \mathrm{~min}$ after injection) each mouse was subjected to anesthesia, perfusion and organs collection. Half of each 
organ was fixed in formalin (10\% neutral buffered formalin solution) until used. The other half was freezed in liquid nitrogen.

The frozen samples were thawed, equilibrated to room-temperature, homogenized in PBS $+0.1 \%$ Triton X-100 (Ratio 75:1 V/W), spun at 10,000 g, $10 \mathrm{~min}$ at $4^{\circ} \mathrm{C}$. The Fluorescein in the homogenized samples, was excited at a wavelength of $494 \mathrm{~nm}$ and its emission intensity was measured at $521 \mathrm{~nm}$. Samples were tested undiluted in duplicates, with Fluorescence Plate Reader, Fluoroskan Ascent FL, Labsystems, Thermo scientific.

The formalin fixed tumors were embedded and sliced at PATHO-LAB Diagmostic, Rehovot, Israel. Photos were taken with a Leica SP8 Confocal Laser Scanning Microscope at The Robert H. Smith Faculty of Agriculture, Food and Environment, The Hebrew University of Jerusalem, Rehovot, Israel.

All cell growth and viability assays, and mice experiments, as well as the photographs taken were performed at Envigo CRS (Israel) Ltd.

\section{Results}

Using our screening platform, SoAP, we have discovered several peptides that target a few proteins, which are overexpressed on the surface of many cancer cells. The targeted proteins were EGFR (Epidermal Growth Factor Receptor), PDL1 (Programmed death-ligand 1), and cMET (tyrosine-protein kinase Met or hepatocyte growth factor receptor-HGFR). These proteins are commonly overexpressed in Lung, Colorectal and Head and Neck cancers. We have also discovered toxic peptides that interact with eEF2 (eukaryotic Elongation Factor 2), a critical protein involved in protein synthesis. In some cases, we used a pro-apoptotic peptide, BIM (a part of the pro apoptotic protein BIM), which is known to be toxic to cells by damaging the mitochondria [10].

\subsection{Cell Growth and Viability Experiments: Mono-Targeting versus Multi-Targeting}

Figure 4 shows the influence of several MuTaTo-like constructs on the growth and viability of A549 cancer cells (human epithelial lung carcinoma; overexpress EGFR, PDL1, cMet) after 48 hours of incubation. The results are shown by photographs (Figure 4(a)), and by Alamar Blue assay (Figure 4(b)). A concentration depended dose response can be seen. Control cells that were treated with PBS are typically crowded and long. The affected cells are much lower in number, and smaller with round shape, or debris. At the concentrations tested $(0.01-1 \mu \mathrm{M})$ no killing effect can be seen with constructs that contained only toxic peptides (PEG-BIM) or one kind of toxic peptide plus one kind of targeting peptide (PEG-E13.3-BIM, PEG-GR-BIM). The same results were with cMet targeting peptide M582 (data not shown). When 2 kinds of targeting peptides were conjugated together with toxic peptides (PEG-E13.3-GR-BIM) a significant decrease in the growth and viability of the cells can be seen at $1 \mu \mathrm{M}$. A similar decrease in the growth and viability of the cells can be seen at a concentration of $0.1 \mu \mathrm{M}$ with 3 targeting peptides 


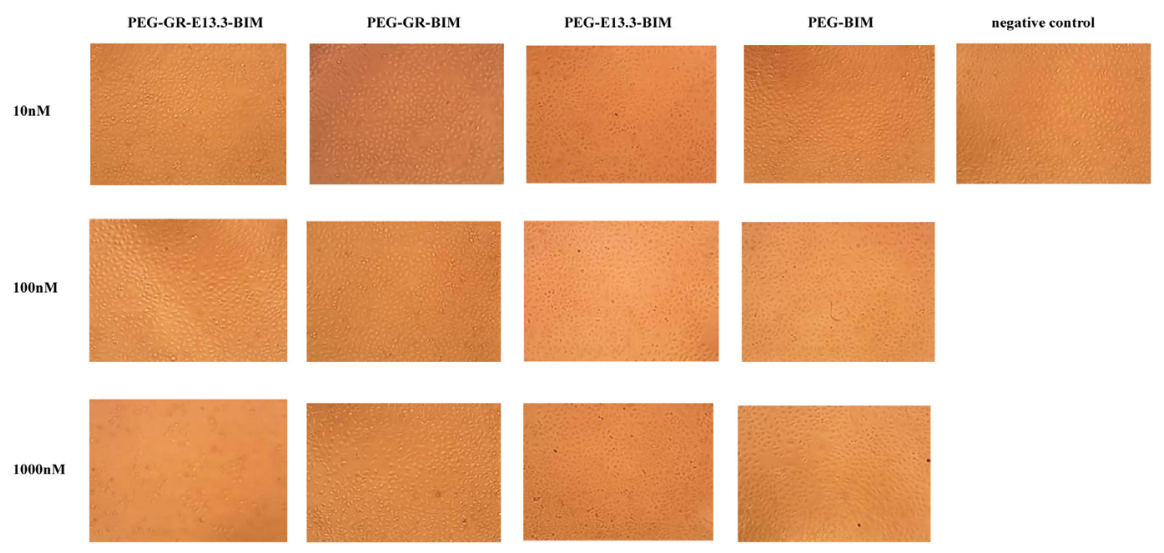

(a)

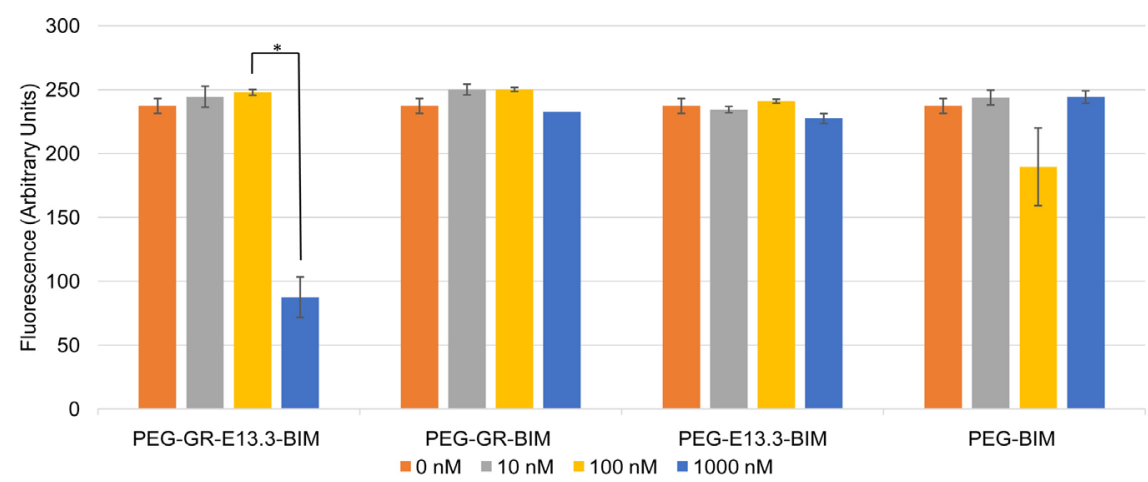

(b)

Figure 4. Cell growth and viability assay of A549 cancer cell line that overexpresses EGFR and PDL1. Cells were incubated for 48 hours with different concentrations of 4 MuTaTo-like constructs. Each construct contained a different combination of peptides, as indicated. E13.3: EGFR-targeting peptide. GR: PDL1-targeting peptide. BIM: toxic peptide. (a) Photographs taken at the end of experiment. Magnification $100 \times$. (b) Alamar Blue assay. ${ }^{\star} \mathrm{P}=$ 0.0049 .

(PEG-E13.3-GR-M582-BIM, Figure 5(a); (PEG-E13.3-GR-M582-GW2, Figure $5(b))$. The above results demonstrate that increasing the number of interactions between our constructs and the cancer cells increases the efficacy of the constructs.

\subsection{Cell Growth and Viability Experiments: High versus Low Expression of Cancer-Targets}

The same principal as above works also when comparing two cancer cell lines that contain different number of cancer targets on their surface, i.e.: the more receptors on the cells, the more effective interaction with the drug will occur. To test this hypothesis, we used the construct PEG-E13.3-BIM that targets EGFR. We tested its influence on the growth and viability of two cancer cell lines: A431 that express 2,000,000 receptors on each cell, and MCF-7 that express 3000 receptors on each cell [11].

Figure 6(a) shows that there is a killing effect of A431 cell line at concentration of $8 \mu \mathrm{M}$, but in MCF-7 this effect is much smaller. Quantification of this 


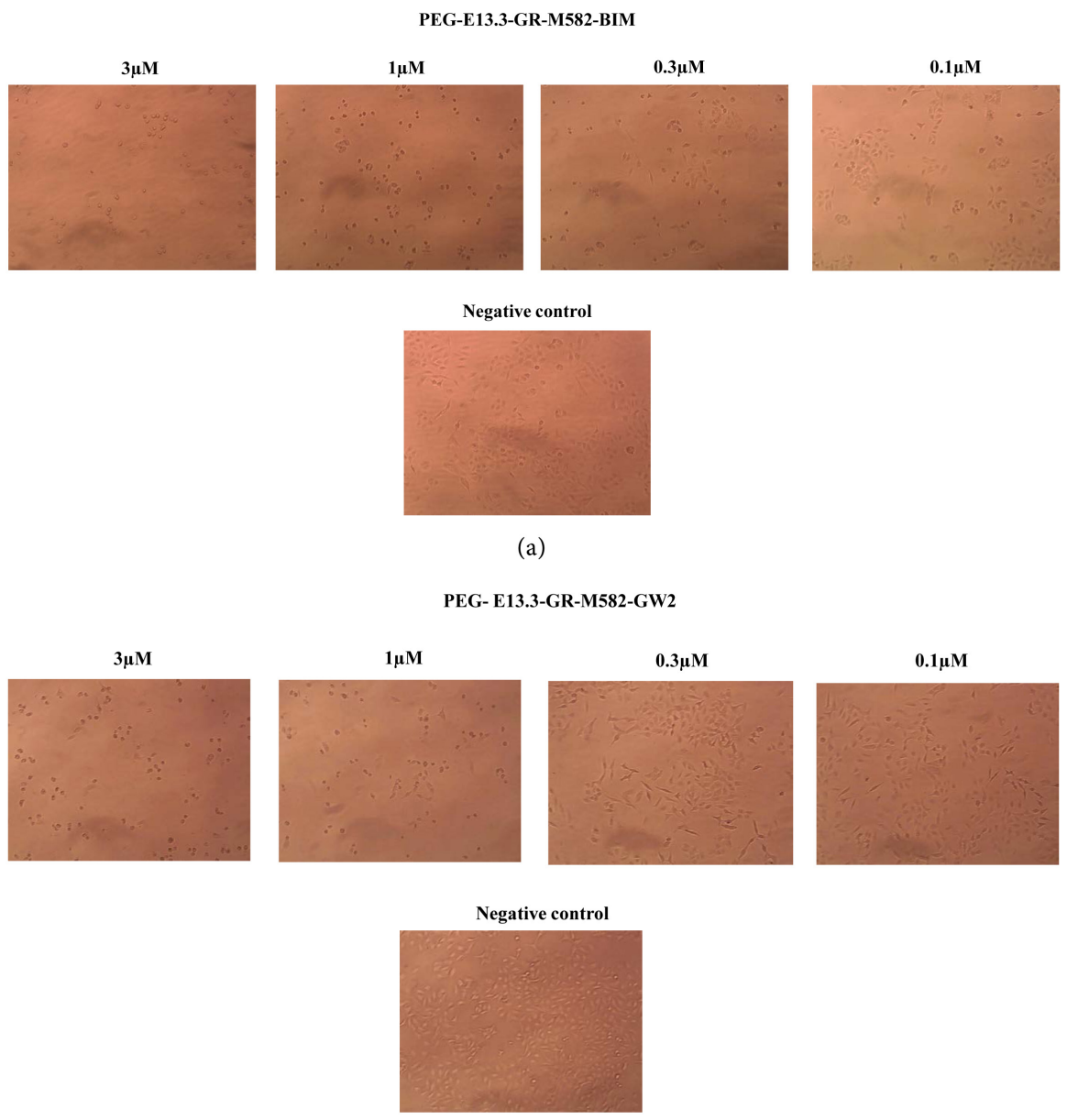

(b)

Figure 5. Cell growth and viability assay of A549 cancer cell line that overexpresses EGFR, PDL1, and cMet. Cells were incubated for 48 hours with different concentrations of MuTaTos. Each construct contained a different combination of peptides, as indicated. E13.3: EGFR-targeting peptide. GR: PDL1-targeting peptide. M582: cMet targeting peptide. BIM, GW2: toxic peptides. Photographs were taken at the end of experiment. Magnification 100×. (a) MuTaTo containing the toxin BIM (targets mitochondria). (b) MuTaTo containing the toxin GW2 (targets eEF2).

effect with Alamar blue assay (Figure 6(b)) showed that the growth and viability of A431 treated with $8 \mu \mathrm{M}$ PEG-E13.3-BIM was $78 \%$ lower than control cells treated with PBS, while the growth and viability of MCF-7 treated with $8 \mu \mathrm{M}$ PEG-E13.3-BIM was 34\% lower than control cells treated with PBS. PEG-BIM (no targeting peptide) had no killing effect with both cell lines (data not shown).

Figure 7 shows a similar experiment, where the toxin BIM was replaced with TOX (a combination of two toxic peptides that target eEF2). The construct PEG-E13.3-Tox gave a killing effect even at $3 \mu \mathrm{M}$ on A431 cells, but much smaller effect on MCF-7 cells even at $8 \mu \mathrm{M}$.

\subsection{Pharmacokinetic (PK) Experiment in Mice}

A simplified MuTaTo model (An EGFR-targeting peptide, E13.3, attached to 

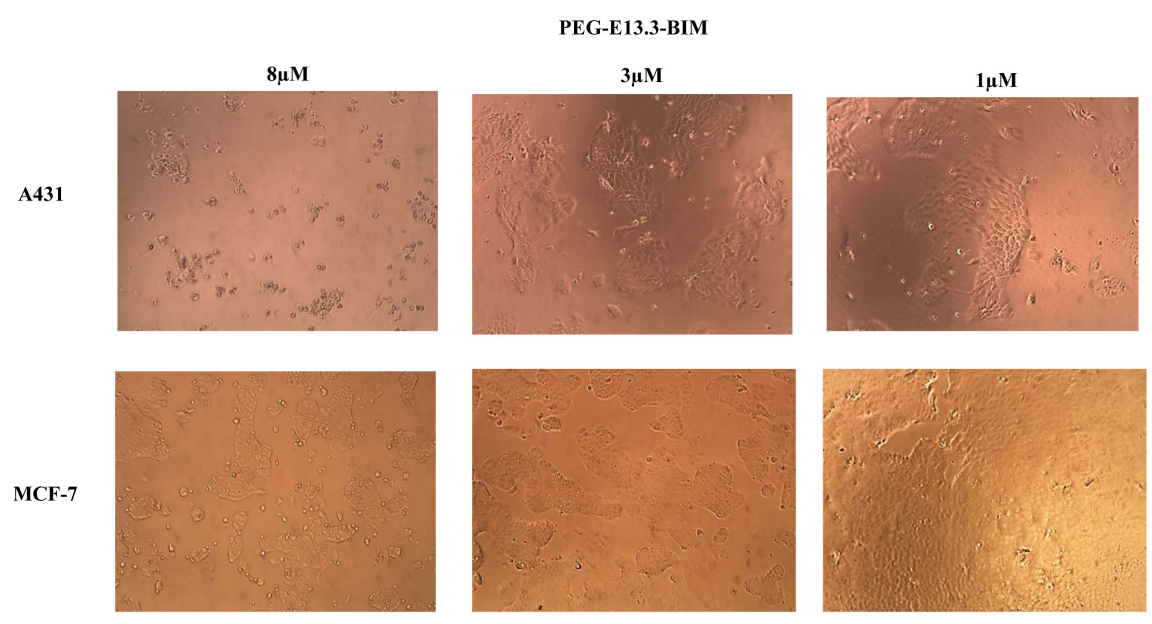

(a)

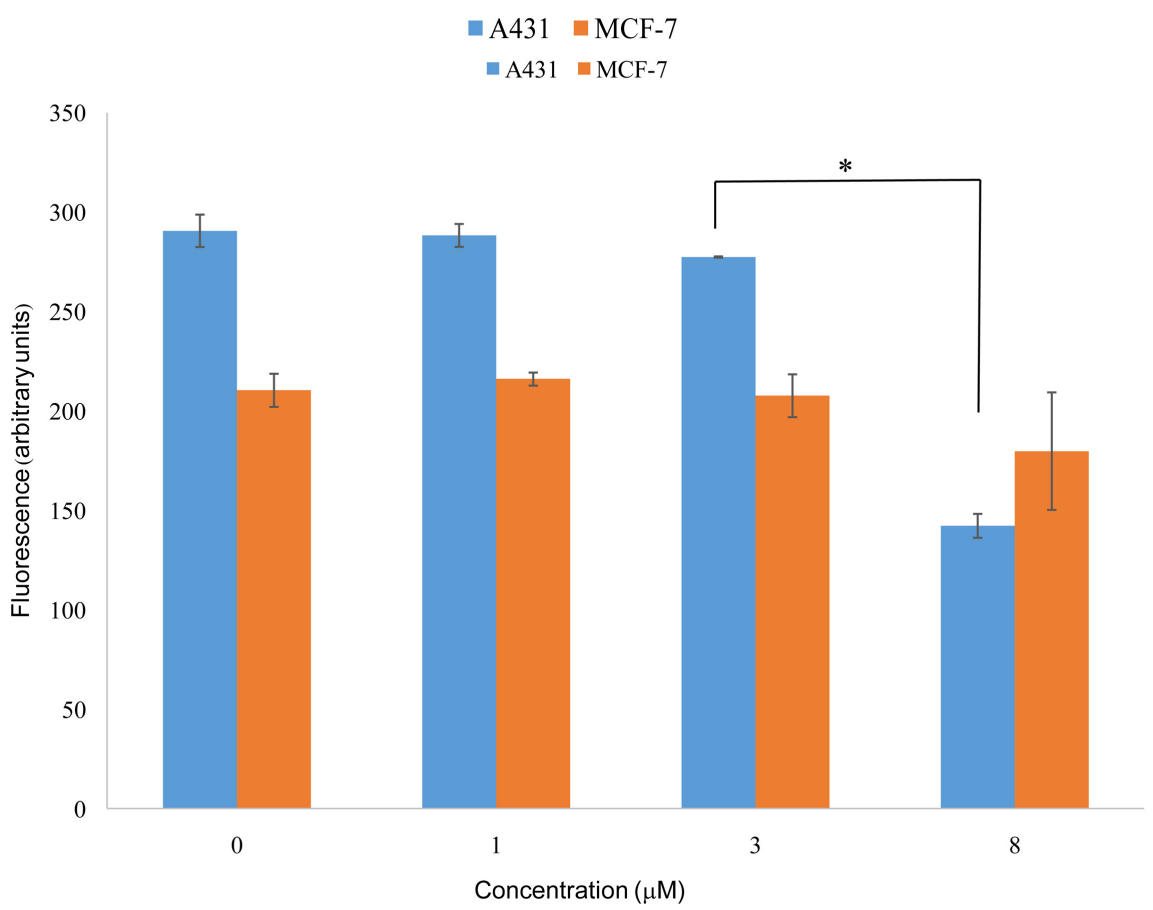

(b)

Figure 6. Cell growth and viability assay of two cell lines that overexpresses different levels of EGFR: A431-very high expression. MCF7-high expression. Cells were incubated for 48 hours with different concentrations of MuTaTo-like construct that contained the peptide E13.3 (targets EGFR), and the toxin BIM. (a) Photographs taken at the end of experiment. Magnification 100×. (b) Alamar Blue assay. ${ }^{\star} \mathrm{P}<0.0001$.

8-arm-PEG) labeled with fluorescein was injected intravenously to Xenograft mice bearing subcutaneous NCI-H1650 tumors (lung cancer that overexpresses EGFR). We followed the construct after the mice were subjected to anesthesia, perfusion and organ collection at different time points. The organs (kidney, liver and tumor) were homogenized, spun and Fluorescence of each sample was measured.

As can be seen in Figure 8(a), the level of fluorescence rapidly increased after 
PEG-E13.3-Tox
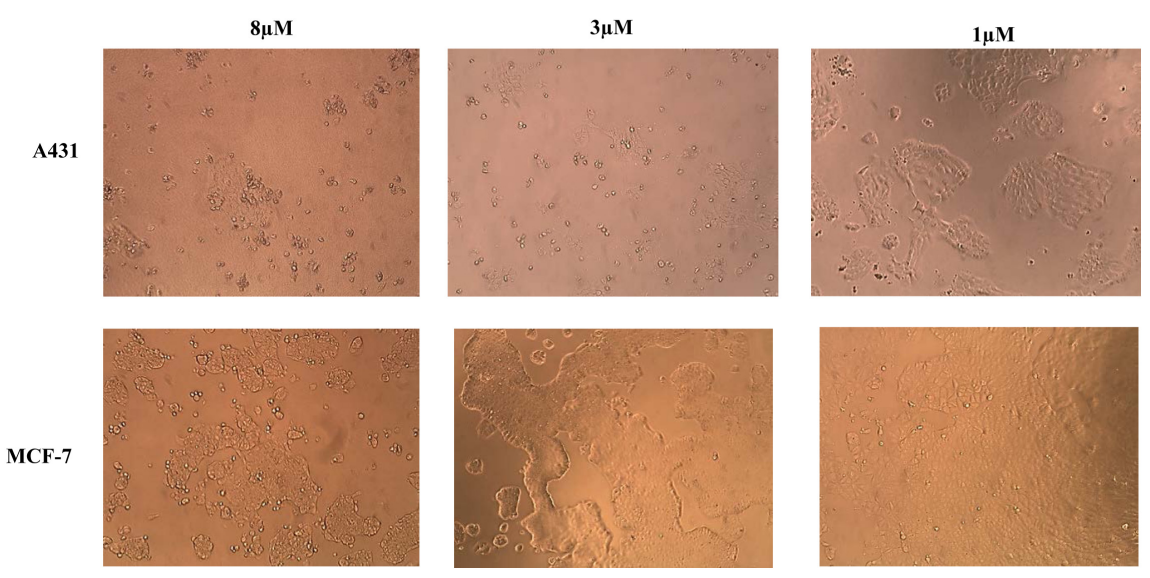

Figure 7. Cell growth and viability assay of two cell lines that overexpresses different levels of EGFR: A431 - very high expression. MCF7—high expression. Cells were incubated for 48 hours with different concentrations of MuTaTo-like construct that contained the peptide E13.3 (targets EGFR), and two toxins (Tox). Photographs taken at the end of experiment. Magnification 100×.

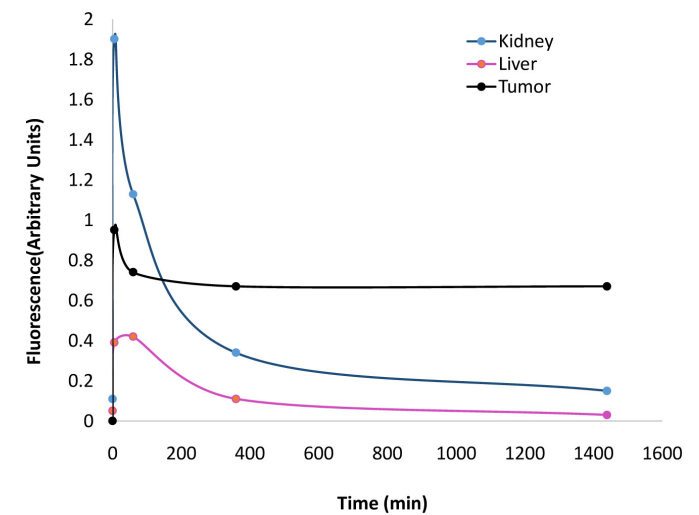

(a)

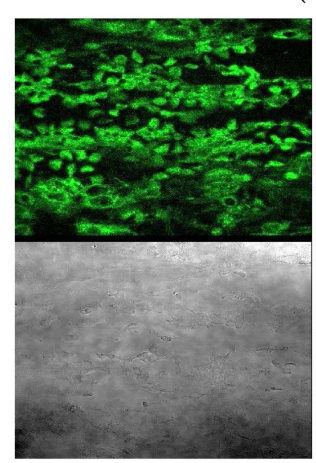

1hr

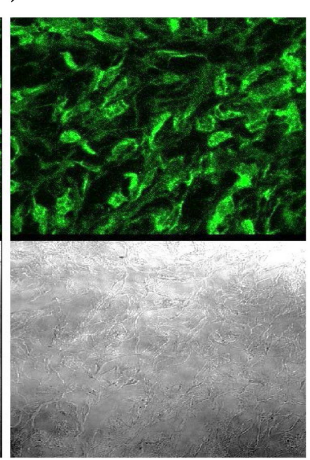

$24 \mathrm{hr}$

(b)

Figure 8. (a) Pharmacokinetic (PK) experiment in mice. Fluorescence level in mice's organs after intravenous injection (IV) of MuTaTo-like construct, that contained fluorescently labeled E13.3 (EGFR-targeting peptide). The tumors were subcutaneous, and contained NCI-H1650 cancer cells that overexpress EGFR. (b) Photographs of tumor-slices taken 1hour or 24 hours after injection, using a confocal fluorescence microscope. Lower photos are of the same subjects, without excitation of fluorescence. Magnification 100×. 
the injection, and then gradually decreased to the original level in the kidneys and livers, while in the tumors the fluorescence was retained 24 hours after the injection. Figure 8(b) shows in the upper part a fluorescent cross-sections of the tumors 1 hour (on the left), and 24 hours (on the right) after the injection, examined under a confocal fluorescence microscope. The lower part is a bright field illumination photograph (visible range) of the same cells.

It can be seen that most of the cells in the tumor interacted with and internalized the fluorescent MuTaTo-like molecules.

\subsection{Xenografts Mice Experiment: The Influence of MuTaTo on Tumor Growth}

Figure 9 describes the results of a mice xenografts experiment with MuTaTo. The xenografts were composed of A549, a human epithelial lung carcinoma that overexpresses EGFR, PD-L1 and cMet receptors. Two 6 mice groups were injected IV after the tumors were established. One group received a negative control of PBS. The other group received A 56-arm MuTaTo that contained 7 copies of E13.3 (anti-EGFR peptide), 7 copies of GR (anti-PD-L1 peptide), 7 copies of M582 (anti-cMet peptide), and 35 copies of the toxic peptide BIM. The dose given was $36 \mathrm{mg} / \mathrm{kg}$ (equivalent to the killing concentration in the in vitro experiment of the same batch; data not shown). The results show that the specific MuTaTo inhibited the growth of the cancer tumors by $42 \%$ at the end of the experiment. No health deterioration was observed with any of the mice.

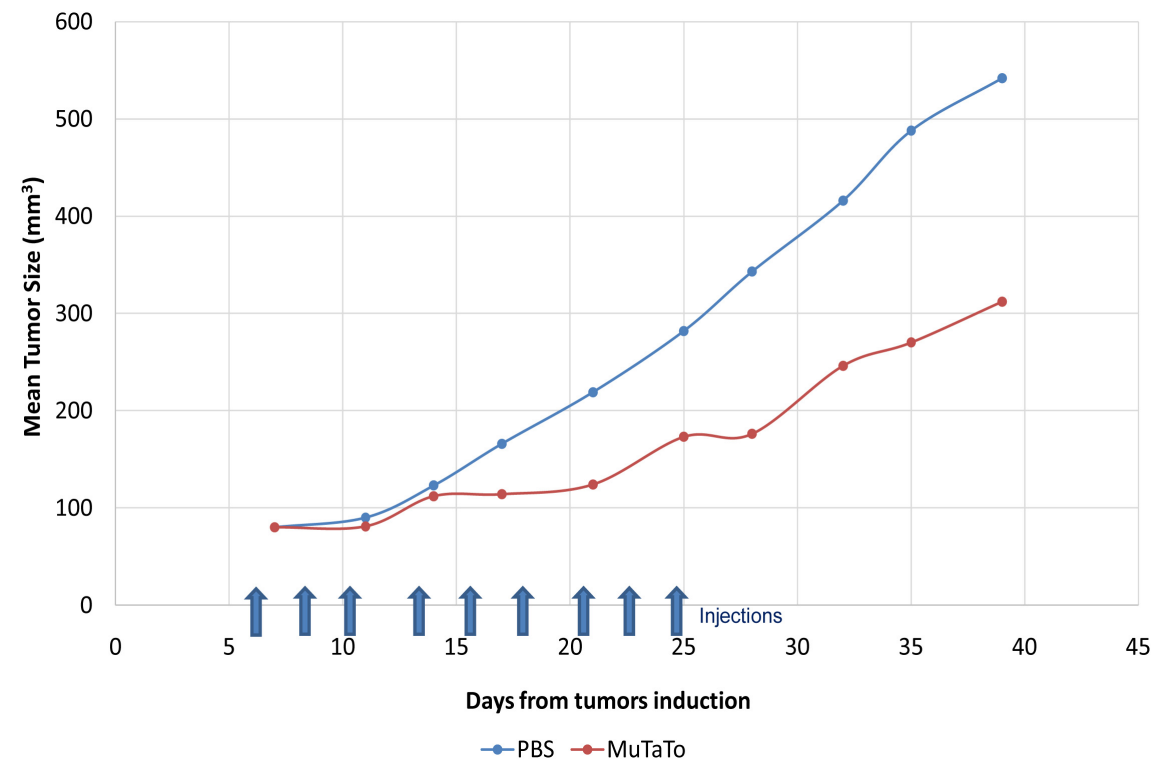

Figure 9. Mice xenograft experiment with MuTaTo. Subcutaneous tumors containing A549 lung cancer cells (overexpress EGFR, PDL1, cMet) were induced in 12 mice. 6 mice were injected IV as indicated with MuTaTo that contained targeting peptides to EGFR, PDL1, cMet, and a toxic peptide (E13.3, GR, M582, BIM respectively). 6 mice served as a control group, and were injected with PBS. Each point represents the mean tumor volume of 5 mice. One exceptional control mouse, and one exceptional MuTaTo treated mouse were excluded. The last 4 data points are statistically different by $\mathrm{T}$ test $(\mathrm{P}<0.05)$. 


\section{Discussion}

In this paper we present $\mathrm{MuTaTo}^{\odot}$, a new personalized medicine concept for curing cancer patients. In the development of this concept we have tried to address all of the factors that cause poor success rate in cancer treatment. We have designed experiments, that would show that the principles behind MuTaTo are sustainable.

Figures 4-7 show that MuTaTo could inhibit the growth of various cancer cells, and in the right concentration could even annihilate them. MuTaTo can also inhibit the growth of established tumors in mice, and hopefully using the right dose would annihilate the tumors.

Figure 2 illustrates how multi-targeting would fight drug-resistance caused by mutations that are acquired by the cancer cells. The optimal number of cancer-targets that should be attacked simultaneously is to be determined. We have chosen to start with three, based on the experience accumulated in years of AIDS treatments with various cocktails. In most cases these cocktails contain three drugs, and these treatments give excellent prognosis [8].

The idea of using conjugates of targeting and cytotoxic moieties is well established, especially in antibody-drug conjugates (ADCs) [12]. The addition of a strong toxin improves the overall efficacy of many drugs. The conjugate should be strong enough to kill all the targeted cancer cells before some of them acquire mutations that would make them drug-resistant. The use of toxic peptides in MuTaTo constructs enables a simple chemistry of production, with only two kinds of starting materials: multi-arm scaffold (in most cases we have used PEG) and small peptides. In addition, all of the peptides used in MuTaTos are hydrophilic, a property that would decrease substantially their ability to penetrate any cell by themselves, in case they are disconnected. This would lower the probability of harming non-cancer cells, and therefore decrease adverse-effects.

The fact that the toxic peptides and the targeting peptides in MuTaTo aim at different pathways gives another benefit. Cancer cells that contain mutations in genes that are downstream in the signal transduction pathway of the targeted receptors would not inhibit the activity of the toxic peptides, and therefore would not acquire drug-resistance due to these mutations. An example for such a situation is a mutation in the gene ras. Since RAS is on the same pathway as EGFR, cells that contain such a mutation would not benefit from an anti-EGFR antibody treatment [6]. On the contrary to this situation, as shown in Figure 4 and Figure 5, A549 cells, which contain a ras mutation, are well treated by a MuTaTo-like construct that contains only EGFR-targeting peptides and toxic peptides.

Each toxic peptide has a specific target inside the cell. Mutations in these targets could help the cancer cells escape from the poisoning effect of the toxic peptides. It would be favorable to use several kinds of toxic peptides in each MuTaTo, to decrease the probability of the cancer cells to escape death by preexisting or new acquired mutations. 
MuTaTo constructs are big. The 56-arm MuTaTos we have used had a molecular weight around $200 \mathrm{kD}$. The 8 -arm MuTaTos we have used had a molecular weight around $100 \mathrm{kD}$. The size of the MuTaTos is bigger than the kidneys cutoff [13]. This increases the half-life of these constructs comparing to the peptides they contain. We have improved the half-life in mice blood from 4 minutes in the case of one peptide to 3 hours when 8 copies of the same peptide were connected to 8-arm PEG (data not shown). The peptides that are connected to PEG are also partly protected from digestion with an increase in their metabolic stability in serum [14]. Although they are big, they are very flexible and hydrophilic due to the long PEG arms (Figure 1). Their flexibility would enable the penetration of MuTaTos deep into solid tumors as shown in Figure 8(b). Another benefit is that MuTaTos, which are peptide-based, would not bind strongly to the outer layer of solid tumors, a situation that could inhibit the tumor-penetration of high-affinity drugs. It has been suggested that low affinity antibodies penetrate better into solid tumors than high affinity ones [15] [16].

The fact that MuTaTo constructs are flexible with no rigid structure, and contain only small peptides, not only contribute to their ability to penetrate solid tumors, but also to escape immune response that can cause drug depletion comparing to other constructs with similar size, like antibodies [17].

The avidity effect of MuTaTo can increase the therapeutic window in treating cancer, that is to lower the effective dose that kills specific cancer cells, without decreasing the dose that starts influencing non cancer cells. MuTaTo uses multiple targeting-peptides that are connected together to achieve this purpose as shown in Figures 4-7. By increasing the number of potential interactions between a cell and a drug, the effect of the drug upon the cell is increased. These results are in accordance with the rational demonstrated in Figure 3. Using a mixture of the same targeting peptides, which are not connected to each other, as in combination therapy, would not give the avidity effect. In this case the level of the adverse-effects may be decreased due to synergism [18], but not as much as when avidity effect is involved [5]. These facts indicate that using avidity effect in cancer treatments, as in MuTaTo, has a great potential in lowering adverse-effects.

The use of at least three peptides on each MuTaTo would increase its specificity and efficacy towards the targeted cancer cells, comparing to mono-targeting, as shown in Figure 4(a) and Figure 5(a). It would also decrease the probability of the cancer cells to bypass the drug, and develop drug-resistance, by pre-existing or new mutations. For the same reason cancer stem cells, metastases and intratumor genetic variant cancer cells would also be targeted by MuTaTo, because they would probably share some of the targeted receptors as the primary cancer cells [19].

Discovering novel peptides that aim at specific targets is relatively easy and fast. We have been using our screening platform, SoAP, for this purpose. Our goal is to discover around 100 novel peptides that would serve as cancer targeting and toxic peptides. Combinations of these peptides within different MuTaTos would cover most of the cancers. 
In our vision each cancer patient's biopsy would be tested for the expression levels of proteins (mostly receptors) on the cells' surface. This could be done by using the targeting peptides that are used in the various MuTaTos, or by using specific antibodies. A specific MuTaTo would be chosen as a treatment for each patient, based on this analysis. The most common cancers would have MuTaTo drugs as shelf products, and treatments for them would be available immediately. Rare cancers would probably require a small scale production of specific MuTaTos. Based on the aspects that were examined in this article, we believe that the MuTaTo concept would be able to offer the treatment of choice for almost any cancer patient, with a great efficacy, and minimal adverse-effects.

\section{Conflicts of Interest}

The authors declare no conflicts of interest regarding the publication of this paper.

\section{References}

[1] Roth, G.A., et al. (2018) Global, Regional, and National Age-Sex-Specific Mortality for 282 Causes of Death in 195 Countries and Territories, 1980-2017: A Systematic Analysis for the Global Burden of Disease Study 2017. The Lancet, 392, 1736-1788. https://doi.org/10.1016/S0140-6736(18)32203-7

[2] Holohan, C., Van Schaeybroeck, S., Longley, D.B. and Johnston, P.G. (2013) Cancer Drug Resistance: An Evolving Paradigm. Nature Reviews Cancer, 13, 714-726. https://doi.org/10.1038/nrc3599

[3] Schirrmacher, V. (2019) From Chemotherapy to Biological Therapy: A Review of Novel Concepts to Reduce the Side Effects of Systemic Cancer Treatment. International Journal of Oncology, 54, 407-419.

[4] Flaherty, D.K. (2012) Immunology for Pharmacy. In: Immunogenicity and Antigenicity, Elsevier Inc., New York, 23-30.

https://doi.org/10.1016/B978-0-323-06947-2.10003-3

[5] Rudnick, S.I. and Adams, G.P. (2009) Affinity and Avidity in Antibody-Based Tumor Targeting. Cancer Biotherapy and Radiopharmaceuticals, 24, 155-161. https://doi.org/10.1089/cbr.2009.0627

[6] Sorich, M.J., et al. (2015) Extended RAS Mutations and Anti-EGFR Monoclonal Antibody Survival Benefit in Metastatic Colorectal Cancer: A Meta-Analysis of Randomized, Controlled Trials. Annals of Oncology, 26, 13-21. https://doi.org/10.1093/annonc/mdu378

[7] Jeyasekharan, A.D. (2019) JAK-ing up Antibody Delivery to Cancer. Science Translational Medicine, 11, 1720. https://doi.org/10.1126/scitranslmed.aax1720

[8] Lu, D.Y., et al. (2018) HAART in HIV/AIDS Treatments: Future Trends. Infectious Disorders Drug Targets, 18, 15-22. https://doi.org/10.2174/1871526517666170505122800

[9] Kitov, P.I. and Bundle, D.R. (2003) On the Nature of the Multivalency Effect: A Thermodynamic Model. Journal of the American Chemical Society, 125, 16271-1684. https://doi.org/10.1021/ja038223n

[10] Sarosiek, K.A., et al. (2013) BID Preferentially Activates BAK While BIM Preferentially Activates BAX, Affecting Chemotherapy Response. Molecular Cell, 51, 751-765. https://doi.org/10.1016/j.molcel.2013.08.048 
[11] Davidson, N.E., Gelmann, E.P., Lippman, M.E. and Dickson, R.B. (1987) Epidermal Growth Factor Receptor Gene Expression in Estrogen Receptor-Positive and Negative Human Breast Cancer Cell Lines. Molecular Endocrinology, 1, 216-223. https://doi.org/10.1210/mend-1-3-216

[12] Thomas, A., Teicher, B.A. and Hassan, R. (2016) Antibody-Drug Conjugates for Cancer Therapy. The Lancet Oncology, 17, e254-e262. https://doi.org/10.1016/S1470-2045(16)30030-4

[13] Anderson, N.L. and Anderson, N.G. (2002) The Human Plasma Proteome: History, Character, and Diagnostic Prospects. Molecular \& Cellular Proteomics, 1, 845-867. https://doi.org/10.1074/mcp.R200007-MCP200

[14] Tang, Y.C., et al. (2019) Identification, Characterization, and Optimization of Integrin $\alpha_{\mathrm{v}} \beta_{6}$-Targeting Peptides from a One-Bead One-Compound (OBOC) Library: Towards the Development of Positron Emission Tomography (PET) Imaging Agents. Molecules, 24, 309. https://doi.org/10.3390/molecules24020309

[15] Thurber, G.M., Schmidt, M.M. and Wittrup, K.D. (2008) Antibody Tumor Penetration: Transport Opposed by Systemic and Antigen-Mediated Clearance. Advanced Drug Delivery Reviews, 60, 1421-1434. https://doi.org/10.1016/j.addr.2008.04.012

[16] Langmuir, V.K., Mendonca, H.L. and Woo, D.V. (1992) Comparisons between Two Monoclonal Antibodies That Bind to the Same Antigen but Have Differing Affinities: Uptake Kinetics and ${ }^{125}$ I-Antibody Therapy Efficacy in Multiceli Spheroids. Cancer Research, 52, 4728-4734.

[17] Pratt, K.P. (2018) Anti-Drug Antibodies: Emerging Approaches to Predict, Reduce or Reverse Biotherapeutic Immunogenicity. Antibodies, 7, 19. https://doi.org/10.3390/antib7020019

[18] Mokhtari, R.B., et al. (2017) Combination Therapy in Combating Cancer. Oncotarget, 8, 38022-38043. https://doi.org/10.18632/oncotarget.16723

[19] Schmitt, M.W., Loeb, L.A. and Salk, J.J. (2016) The Influence of Subclonal Resistance Mutations on Targeted Cancer Therapy. Nature Reviews Clinical Oncology, 13, 335-347. https://doi.org/10.1038/nrclinonc.2015.175 